\title{
COMPORTAMIENTO DE UN MATERIAL GRANULAR NO TRATADO EN ENSAYOS TRIAXIALES CÍCLICOS CON PRESIÓN DE CONFINAMIENTO CONSTANTE Y VARIABLE
}

\section{BEHAVIOR OF AN UNBOUND GRANULAR MATERIAL IN CYCLIC TRIAXIAL TESTS WITH CONSTANT AND VARIABLE CONFINING PRESSURE}

\author{
Hugo Alexander Rondón Quintana ${ }^{1} \quad$ Torsten Wichtmann $^{2}$ \\ Theodoros Triantafyllidis ${ }^{3} \quad$ Arcesio Lizcano Peláez ${ }^{4}$ \\ Recibido 5 de junio de 2008, aceptado 3 de octubre de 2008 \\ Received: June 5, 2008 Accepted: October 3, 2008
}

\begin{abstract}
RESUMEN
En un pavimento, cada una de las capas de la estructura experimenta bajo una carga vehicular, ciclos de esfuerzo con componentes vertical, horizontal y de corte. Para el estudio de materiales granulares no tratados (utilizados para conformar capas de base y subbase), la mayor parte de las investigaciones se realizan empleando equipos triaxiales cíclicos en donde sólo la carga vertical es cíclica y la presión de confinamiento permanece constante durante el ensayo. Un ensayo que reproduce mejor la forma como se distribuyen los esfuerzos en estas capas es el ensayo triaxial cíclico con presión de confinamiento variable. En este ensayo se pueden modelar las componentes cíclicas tanto en el sentido vertical como horizontal. A pesar que son ensayos distintos, la ingeniería de pavimentos supone que la respuesta que experimentan estos materiales en estos ensayos es similar, lo anterior basado en algunos estudios realizados en la década de los setenta. En la presente investigación se diseña y desarrolla un programa experimental más detallado, para comparar el comportamiento que desarrolla un material granular no tratado en estos ensayos. De los resultados se evidencia que sólo para algunas trayectorias de esfuerzo, la dirección y la acumulación de la deformación vertical y volumétrica es similar.
\end{abstract}

Palabras clave: Materiales granulares no tratados, deformación permanente, ensayos triaxiales cíclicos.

\begin{abstract}
In a pavement structure, passing wheel loads impose cyclic stresses consisting of vertical, horizontal and shear components. Studies of the behavior of unbound granular materials (UGM, used for base and sub-base layers) under cyclic loading are mostly performed using the axisymmetric triaxial test with constant confining pressure (CCP test) and a cyclic variation of the axial stress. However, in this type of test only the vertical component of the cyclic stress path is considered. The oscillation of the horizontal stress can be reproduced by an additional cyclic variation of the confining pressure (VCP test). $C C P$ and VCP tests are sometimes assumed to deliver similar residual and resilient deformations as long as the average stress is the same in both types of tests. However, this assumption is based on limited test data in the literature. The present paper documents a more detailed comparative study with CCP and VCP tests. The results show that only for some special stress paths both types of test deliver similar permanent axial or volumetric deformations.
\end{abstract}

Keywords: Unbound granular materials, permanent deformation, cyclic triaxial tests.

1 Departamento de Ingeniería Civil. Universidad de los Andes. Carrera 1. Este No 19 A-40, Edificio Mario Laserna, Piso 6, Bogotá D.C. Colombia. E-mail: h-rondon@uniandes.edu.co

2 Institute of Soil Mechanics and Rock Mechanics. University of Karlsruhe. Engler-Bunte-Ring 14, 76131. Karlsruhe, Germany. E-mail: Torsten. Wichtmann@ibf.uni-karlsruhe.de

3 Institute of Soil Mechanics and Rock Mechanics. University of Karlsruhe. Engler-Bunte-Ring 14, 76131. Karlsruhe, Germany. E-mail: Triantafyllidis@ ibf.uni-karlsruhe.de

4 Departamento de Ingeniería Civil. Universidad de los Andes. Carrera 1. Este No 19 A-40, Edificio Mario Laserna, Piso 6. Bogotá D.C., Colombia. E-mail: alizcano@uniandes.edu.co 


\section{INTRODUCCIÓN}

Un pavimento debe ser diseñado de tal manera que las cargas impuestas por el tránsito no generen deformaciones permanentes excesivas. En el caso de los pavimentos flexibles estas deformaciones se producen en cada una de las capas. Los métodos de diseño de pavimentos suponen que la mayor parte de la acumulación de la deformación permanente ocurre en la subrasante. Sin embargo, en vías donde se construyen capas asfálticas delgadas o de baja rigidez (p.e., vías de bajo tráfico) las capas granulares de base y subbase (compuestas por materiales granulares no tratados) soportan el esfuerzo aplicado casi en su totalidad y la magnitud de dichos esfuerzos puede llegar a generar valores elevados de deformación permanente. Adicionalmente diversas investigaciones realizadas sobre pistas de pruebas han demostrado que gran parte de la deformación permanente se produce en las capas granulares [1-10]. Si a lo anterior se suma que la tendencia del parque automotor en Colombia y el mundo en los últimos 30 años ha sido incrementar en número y magnitud de cargas, es evidente entonces pensar que como criterio de diseño, las metodologías empíricas o mecanicistas deben darle mayor importancia a las deformaciones que se producen en estas capas. Por otro lado, la forma para caracterizar y evaluar de manera indirecta la "calidad" de materiales granulares para base y subbase en un pavimento es por medio de la realización de ensayos empíricos, los cuales no pueden predecir la rigidez y la resistencia a la deformación permanente que experimentan bajo una carga rodante (carga cíclica).

Por lo anterior, la ingeniería de pavimentos ha venido desarrollando estudios para intentar comprender el comportamiento elastoplástico que experimentan materiales granulares bajo diversas trayectorias de cargas cíclicas

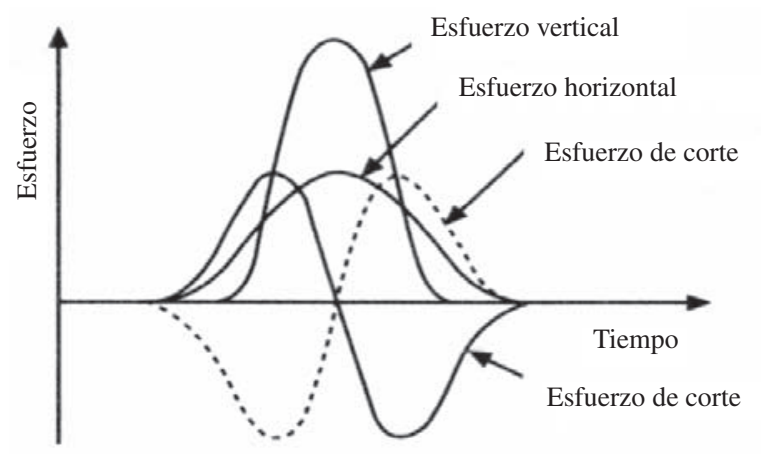

Figura 1. Variación de esfuerzos con respecto al tiempo cuando se aplica una carga vehicular a un pavimento. y condiciones del medio ambiente. El estado actual del conocimiento en esta área es que el comportamiento de estos materiales aún no ha sido totalmente entendido [5, 11-17].

Una de las principales limitaciones para estudiar la respuesta que experimentan materiales granulares en un pavimento ha sido la carencia de equipos apropiados para caracterizar su respuesta dinámica. Actualmente no existen equipos de laboratorio que puedan medir simultáneamente la influencia que tienen factores tales como la magnitud de las cargas que se mueven sobre el pavimento y la evolución en el tiempo de las condiciones ambientales. La mayor parte de las investigaciones sobre materiales granulares en el área de los pavimentos se realizan por medio de ensayos triaxiales cíclicos con presión de confinamiento constante (PCC). En este ensayo sólo se puede aplicar esfuerzo vertical cíclico a una muestra, y en un pavimento el esfuerzo presenta componentes vertical, horizontal y de corte (figura 1).

En comparación con los ensayos PCC, los ensayos triaxiales cíclicos con presión de confinamiento variable (PCV) simulan mejor el comportamiento que experimenta un material granular en un pavimento, ya que puede realizar ciclos de carga en las direcciones vertical y horizontal. A pesar que el material granular en estos dos ensayos experimenta trayectorias de esfuerzo y por lo tanto comportamiento totalmente diferente, la ingeniería de pavimentos supone que la respuesta de materiales granulares (rigidez y deformación permanente) en ambos ensayos es la misma. La anterior afirmación se basa principalmente en los estudios realizados por Allen y Thompson [18], Brown [19] y Brown y Hyde [20]. Estos últimos emplearon las trayectorias de esfuerzos que se presentan en la figura 2 para comparar el comportamiento del material granular

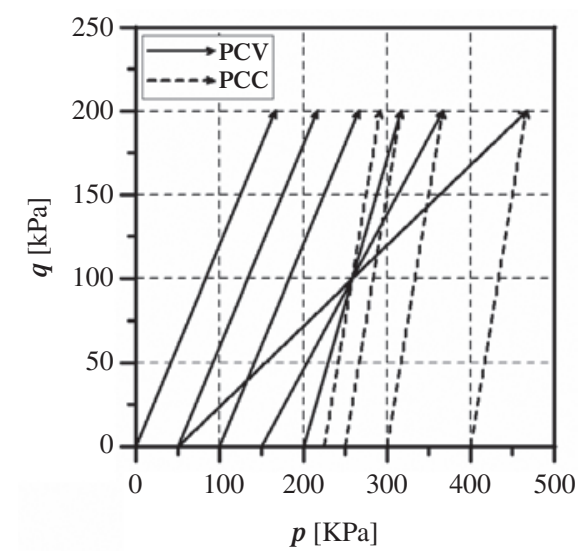

Figura 2. Trayectorias de esfuerzo utilizadas por Brown y Hyde [20]. 
en ensayos PCC y PCV ( $q$ es esfuerzo desviador y $p$ es la presión media ejercida).

Brown y Hyde [20] reportaron valores similares de deformación permanente en ambos ensayos (figura 3). La principal limitación de este estudio fue que no se permitió evaluar la influencia del esfuerzo desviador máximo $q_{\max }$ (ya que éste fue siempre de $200 \mathrm{kPa}$ ) y de la inclinación de las trayectorias de esfuerzo sobre el comportamiento de los materiales ensayados (ver figura 2).

Uno de los objetivos de este proyecto de investigación fue, entonces, comparar la respuesta que experimenta un material granular tipo base BG-2 (de acuerdo a [21]) en estos dos tipos de ensayos. Para tal fin se diseñó una fase experimental que tuvo en cuenta los alcances y las limitaciones presentadas en estudios similares (principalmente los de [18-20]).

Algunos de los modelos matemáticos desarrollados para simular la respuesta que experimentan estos materiales granulares no tratados bajo carga cíclica, los factores que afectan dicha respuesta y un estado del conocimiento detallado sobre el tema pueden ser consultados en [13, 22-24].

\section{DESARROLLO EXPERIMENTAL}

La fase experimental se divide en dos etapas. La primera corresponde a los ensayos de caracterización y resistencia mecánica del material bajo carga monotónica. En una segunda fase se realizaron los ensayos triaxiales cíclicos

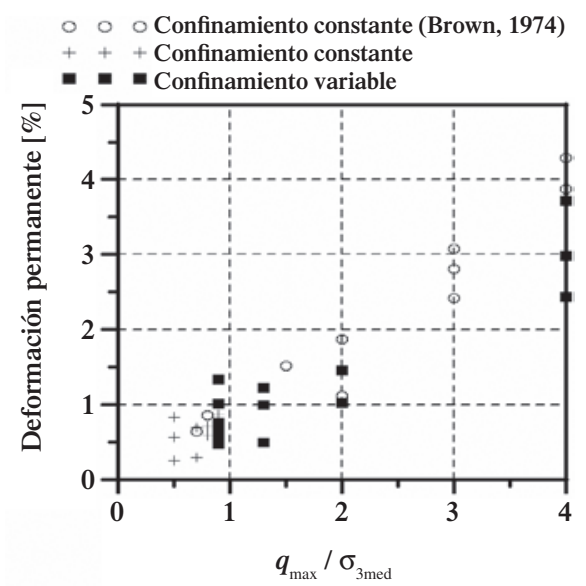

Figura 3. Deformación permanente vs. la relación entre el esfuerzo desviador máximo y la presión de confinamiento $q_{\max } / \sigma_{3 \mathrm{med}}$. drenados tipo PCC y PCV para medir y evaluar el comportamiento que experimenta el material granular en estos ensayos. Todos los ensayos fueron realizados en los laboratorios del Instituto de Mecánica de Suelos y de Cimentaciones de la Ruhr Universität Bochum en Alemania.

A continuación se mencionan los ensayos de caracterización realizados al material, los cuales en su gran mayoría fueron ejecutados siguiendo las Normas de Ensayos de Materiales para Carreteras del Instituto Nacional de Vías [25]: Análisis granulométrico de agregados gruesos y finos (INV. E - 213), peso específico (INV. E - 222, 223), porcentaje de partículas fracturadas (INV. E - 227), índice de alargamiento y aplanamiento (INV. E - 230), índice de plasticidad (INV. E - 125, 126), Proctor modificado (INV. E - 142), ángulo de reposo para la estimación del ángulo de fricción crítico, densidad máxima y mínima seca del material $\left(\rho_{\text {dmax }}, \rho_{\text {dmin }}\right.$, respectivamente) de acuerdo a la norma alemana DIN 18126 y triaxial monotónico drenado con el material en estado denso.

\section{Ensayos de caracterización}

El material utilizado para los ensayos está formado básicamente por partículas sub-angulares de cuarzo y es un material típico utilizado como base granular en Alemania. La granulometría utilizada es acorde a la exigida por las especificaciones colombianas para la conformación de bases granulares tipo BG-2 en pavimentos flexibles [21] (figura 4), excepto por el tamaño máximo de partícula $\left(d_{\max }\right)$ requerido.

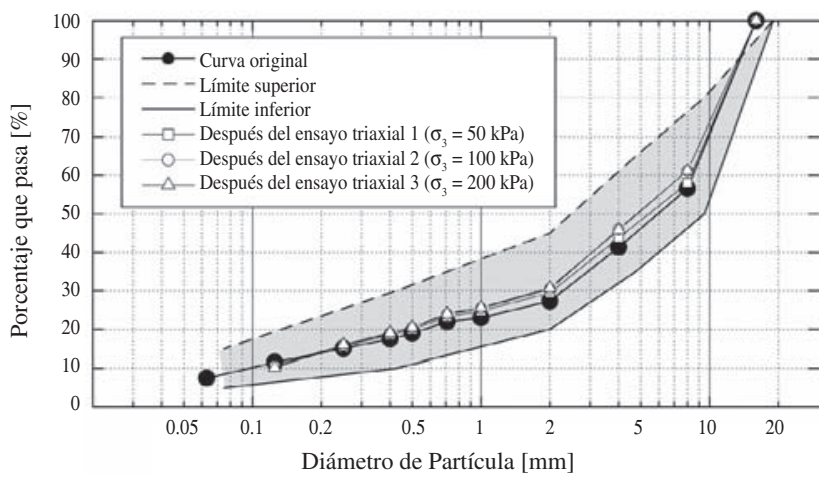

Figura 4. Distribución granulométrica del material (denotado como "curva original") comparado con los valores límites de la especificación INVIAS [21]. 
El $d_{\max }$ se redujo a $16 \mathrm{~mm}$ con el fin de que la relación entre la longitud de la sección transversal $(b)$ de las muestras en el ensayo triaxial y $d_{\max }\left(b / d_{\max }\right)$ fuera mayor a 5 (valor requerido por la especificación alemana DIN 18137 para el caso de materiales bien gradados). El diámetro medio de partícula $\left(d_{50}\right)$ es $6.3 \mathrm{~mm}$ y el coeficiente de uniformidad $\left(C_{\mathrm{u}}=d_{60} / d_{10}\right)$ es 100 . El peso específico del material $\left(\rho_{\mathrm{s}}\right)$ es de $2.65 \mathrm{~g} / \mathrm{cm}^{3}$ y se utilizó un picnómetro para su determinación. Con base en la especificación alemana DIN 18 126, la máxima densidad seca del material $\left(\rho_{\text {dmax }}\right)$ es de $2.16 \mathrm{~g} / \mathrm{cm}^{3}$ (determinada en una mesa vibratoria) y la mínima $\left(\rho_{\text {dmin }}\right)$ de $1.84 \mathrm{~g} / \mathrm{cm}^{3}$. Con los anteriores valores se obtuvieron las relaciones de vacíos mínima $\left(e_{\min }=0.225\right)$ y máxima $\left(e_{\max }=0.444\right)$ del material. El contenido óptimo de agua determinado en el ensayo Proctor fue de $5.2 \%$ y la densidad seca máxima $\left(\rho_{\text {dmaxPr }}\right)$ de $2.30 \mathrm{~g} / \mathrm{cm}^{3}$. Este porcentaje de agua fue el utilizado para la elaboración de las muestras de los ensayos triaxiales monotónicos y cíclicos. El ángulo de fricción crítico $\left(\varphi_{\mathrm{c}} \approx 38^{\circ}\right)$ fue determinado con base en el ensayo de ángulo de reposo con el material en estado seco. La fracción fina del material no presentaba plasticidad, los índices de alargamiento, aplanamiento y caras fracturadas presentaban valores de $29 \%, 33 \%$ y $70 \%$, respectivamente.

\section{Ensayos triaxiales monotónicos}

Tres ensayos triaxiales monotónicos bajo condición drenada con el material saturado en estado denso (índice de densidad relativo $I_{\mathrm{D} 0}=\left(e_{\max }-e\right) /\left(e_{\max }-e_{\min }\right)=1.06-1.13$ y densidad seca $\rho_{d}>95 \%$ de la $\rho_{\text {dmaxPr }}$ ) fueron realizados para determinar los parámetros de resistencia al corte. Los esfuerzos laterales efectivos $\left(\sigma_{3}\right)$ de los tres ensayos

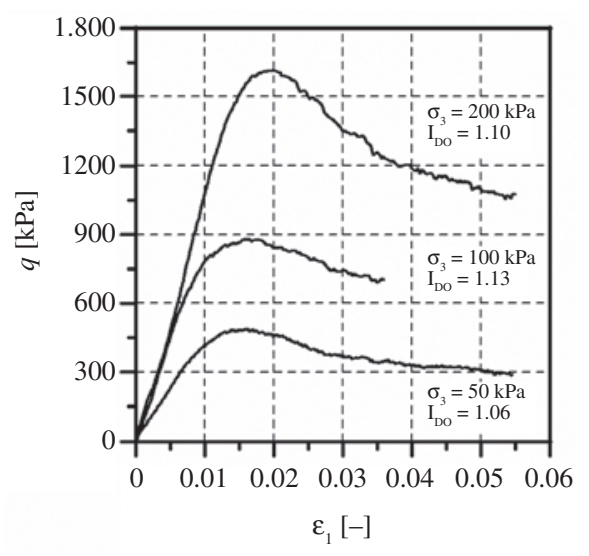

Figura 5. $q$ vs. $\varepsilon_{1}$ en ensayo triaxial con diferentes presiones de confinamiento. fueron $\sigma_{3}=-50,-100$ y $-200 \mathrm{kPa}$ y las muestras presentaban sección transversal cuadrada de $8.7 \mathrm{~cm}$ x $8.7 \mathrm{~cm}$ y altura $h$ de $18 \mathrm{~cm}$. Las muestras fueron preparadas por fuera del equipo triaxial en un molde compuesto de cuatro caras metálicas apoyado sobre la base de carga del triaxial. Una descripción detallada del equipo triaxial utilizado, de la compactación y preparación de las muestras y los equipos utilizados puede ser consultado en [27]. Para compactar las muestras se utilizó un martillo tipo proctor a escala el cual aplicaba un peso $(W)$ de $10 \mathrm{~N}($ masa $=1 \mathrm{~kg})$ y una altura de caída $(H)$ de $20 \mathrm{~cm}$. La altura de $18 \mathrm{~cm}$ de la muestra fue dividida en 6 capas $(s)$ de material granular y a cada capa eran aplicados 250 golpes $(B)$ con el martillo a escala. Aproximadamente se aplicó una energía por volumen $(E)$ de $2200 \mathrm{kNm} / \mathrm{m}^{3}$, acordando a la ecuación empírica (1) del ensayo Proctor.

$$
E=\frac{B \cdot s \cdot W \cdot H}{V}
$$

$\mathrm{V}$ es el volumen de la muestra $\left(1362 \mathrm{~cm}^{3}\right)$. Esta energía fue escogida con el fin de alcanzar densidades entre el 95 y $97 \%$ de la densidad seca máxima del ensayo Proctor modificado.

Después de la compactación, la muestra era colocada en la celda triaxial. Luego, se colocaba la membrana (diámetro de $110 \mathrm{~mm}$ y espesor de $0.6 \mathrm{~mm}$ ) usando un aparato diseñado para tal fin (de sección transversal cuadrada). Las curvas de esfuerzo desviador $\left(q=-\left[\sigma_{1}-\sigma_{3}\right]\right)$ y de deformación volumétrica $\left(\varepsilon_{\mathrm{v}}=\varepsilon_{1}+2 \varepsilon_{3}, \varepsilon_{3}\right.$ es la deformación radial) vs. la deformación vertical $\left(\varepsilon_{1}\right)$ son presentadas en las figuras 5 y 6 , respectivamente.

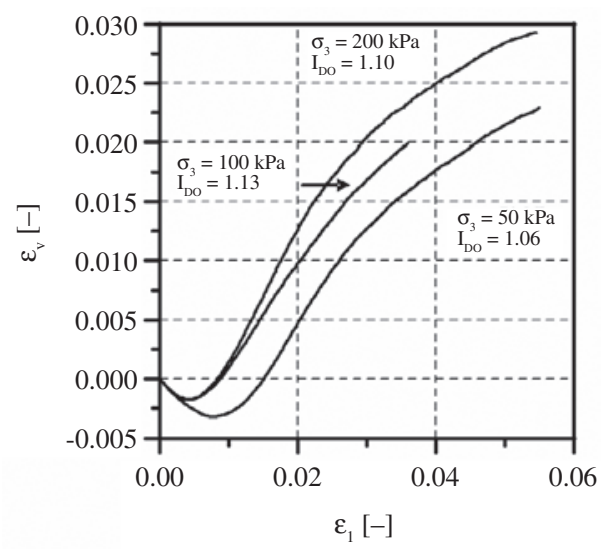

Figura 6. $\varepsilon_{\mathrm{v}}$ vs. $\varepsilon_{1}$ en ensayo triaxial con diferentes presiones de confinamiento. 
En esta investigación los ensayos triaxiales monotónicos y cíclicos se realizaron empleando muestras prismáticas. Sin embargo, la mayor parte de las investigaciones sobre materiales granulares se realiza sobre muestras cilíndricas. Por tal motivo se realizaron siete ensayos triaxiales monotónicos adicionales sobre una fracción de arena del material granular con $d_{50}=0.55 \mathrm{~mm}$ y $C_{\mathrm{u}}=1.8$, para comparar la influencia que tiene la forma de la muestra sobre su comportamiento monotónico. Además, se realizaron ensayos cíclicos sobre la misma muestra de arena con especímenes cilíndricos y prismáticos. Las muestras prismáticas presentaban sección cuadrada de 8.7 x $8.7 \mathrm{~cm}$ y altura de $18 \mathrm{~cm}$ y las cilíndricas un radio de $5 \mathrm{~cm}$ y alturas de 10 y $20 \mathrm{~cm}$. En los ensayos monotónicos cuatro especímenes fueron ensayados con $I_{\mathrm{DO}}=0.55$ 0.58 y los otros tres con $I_{\mathrm{DO}}=0.95-0.99$. En los cíclicos los especimenes fueron ensayados con $I_{\mathrm{DO}}=0.58$. Los resultados de estos ensayos muestran que la influencia de la forma de la muestra sobre el comportamiento del material bajo carga monotónica y cíclica es muy pequeña. Dos ejemplos se presentan en las figuras 7 y 8 . Los resultados de esta parte de la investigación son más detallados en [27-28].

\section{Ensayos triaxiales cíclicos}

En la tabla 1 y en la figura 9 se presenta la información del programa de ensayos triaxiales cíclicos drenados. En esta figura se observa que para cada trayectoria PCV existe una PCC con los mismos valores promedios de esfuerzo desviador, presión media $\left(q_{\text {med }}, p_{\text {med }}\right)$ y esfuerzo desviador máximo $q_{\max }$. A diferencia de los estudios reportados por Brown y Hyde [20], con las trayectorias de esfuerzos presentadas en la figura 9 es posible comparar

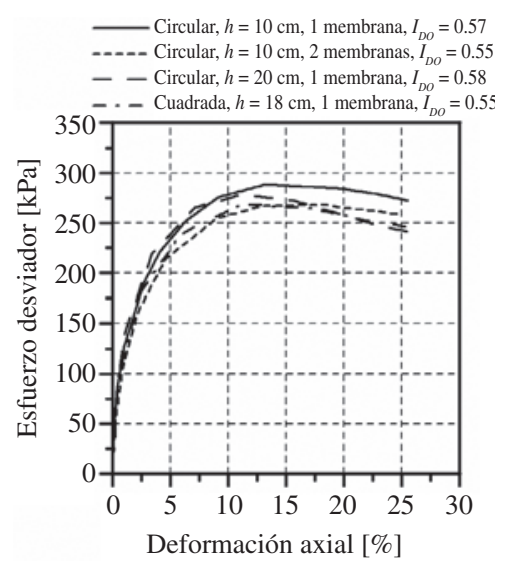

Figura 7. Comparación del comportamiento monotónico de especimenes prismáticos y cilíndricos. $h$ es la altura de la muestra. la influencia de la magnitud del esfuerzo desviador y la pendiente de la trayectoria de esfuerzo $\eta_{\mathrm{ampl}}=q_{\mathrm{ampl}} / p_{\mathrm{ampl}}$ sobre la respuesta que experimentan materiales granulares en ensayos PCC y PCV.

En la figura 10 se describe esquemáticamente la notación de la distribución del esfuerzo durante los ensayos. Al igual que en los ensayos monotónicos las muestras eran saturadas después de ser compactadas empleando el contenido de agua óptimo del ensayo Proctor modificado.

Tabla 1. Programa de ensayos triaxiales cíclicos $\left(p_{\min }=\right.$ presión media mínima, $p_{\max }=$ presión media máxima, $p_{\text {med }}=$ presión media promedio, $p_{\mathrm{ampl}}=$ amplitud de la presión media, $q_{\min }=$ esfuerzo desviador mínimo, $q_{\max }=$ esfuerzo desviador máximo, $q_{\text {med }}=$ esfuerzo desviador promedio, $q_{\text {ampl }}=$ amplitud del esfuerzo desviador.

\begin{tabular}{|cccccccc|}
\hline Ensayo & $\begin{array}{c}\boldsymbol{p}_{\text {min }} \\
{[\mathbf{k P a}]}\end{array}$ & $\begin{array}{c}\boldsymbol{p}_{\text {max }} \\
{[\mathbf{k P a}]}\end{array}$ & $\begin{array}{c}\boldsymbol{p}_{\text {med }} \\
{[\mathbf{k P a}]}\end{array}$ & $\begin{array}{c}\boldsymbol{p}_{\text {ampl }} \\
{[\mathrm{kPa}]}\end{array}$ & $\begin{array}{c}\boldsymbol{q}_{\text {max }} \\
{[\mathbf{k P a}]}\end{array}$ & $\begin{array}{c}\boldsymbol{q}_{\text {med }} \\
{[\mathrm{kPa}]}\end{array}$ & $\begin{array}{c}\boldsymbol{q}_{\text {ampl }} \\
{[\mathbf{k P a}]}\end{array}$ \\
\hline 1 & 20 & 200.0 & 110 & 90 & 135 & 67.5 & 67.5 \\
3 & 20 & 100.0 & 60 & 40 & 60 & 30 & 30 \\
4 & 20 & 100.0 & 60 & 40 & 120 & 60 & 60 \\
5 & 20 & 200.0 & 110 & 90 & 270 & 135 & 135 \\
6 & 20 & 200.0 & 110 & 90 & 202.5 & 101.25 & 101.25 \\
\hline 2,7 & 87.5 & 132.5 & 110 & 22.5 & 135 & 67.5 & 67.5 \\
8 & 50 & 70.0 & 60 & 10 & 60 & 30 & 30 \\
9 & 40 & 80.0 & 60 & 20 & 120 & 60 & 60 \\
10 & 65 & 155.0 & 110 & 45 & 270 & 135 & 135 \\
11 & 76.25 & 143.8 & 110 & 33.78 & 202.5 & 101.25 & 101.25 \\
\hline
\end{tabular}

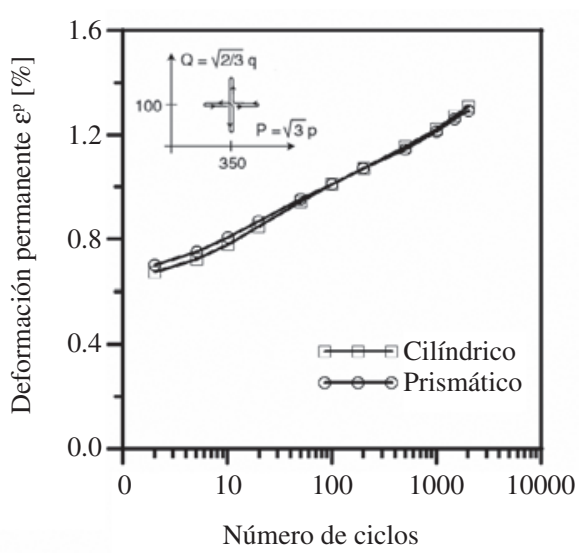

Figura 8. Comparación del comportamiento cíclico de especímenes prismáticos y cilíndricos. 


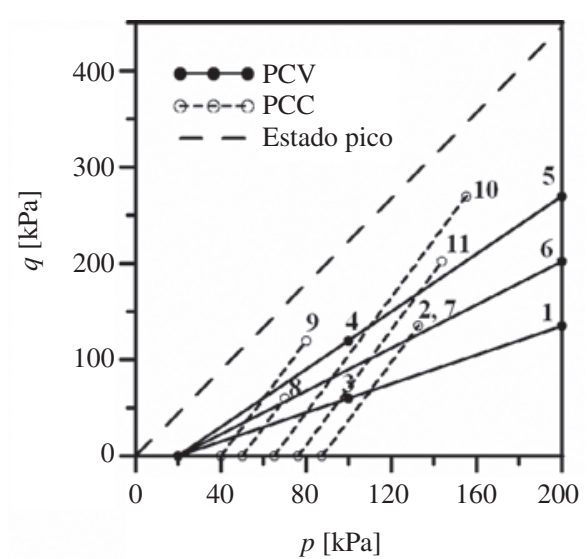

Figura 9. Trayectorias de esfuerzos utilizadas para los ensayos cíclicos.

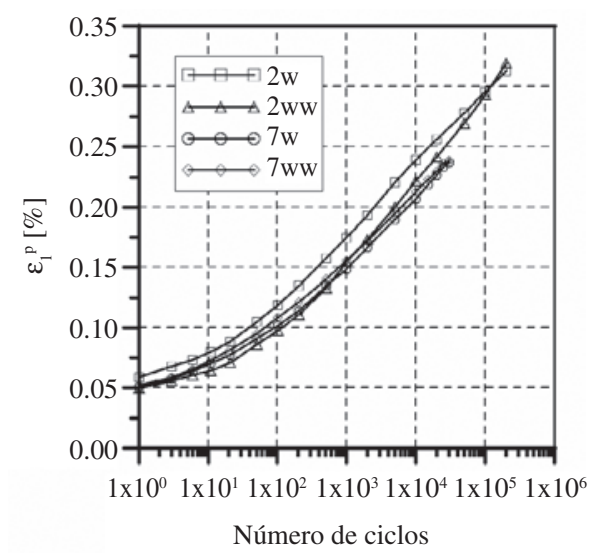

Figura 11. Influencia de la frecuencia de carga $(f)$. Análisis de ensayos PCC.

Las frecuencias de carga $(f)$ fueron de $0.05 \mathrm{~Hz}$ y de $1.0 \mathrm{~Hz}$ para los ensayos PCV y PCC, respectivamente. Esta diferencia en la frecuencia fue debido a razones técnicas de operación del equipo triaxial, ya que la máxima velocidad de carga en el ensayo PCV debe ser de $0.05 \mathrm{~Hz}$. Basados en estudios teóricos y experimentales, diferentes investigadores han reportado que la influencia de la frecuencia de carga sobre las características resilientes y de deformación permanente en arenas y materiales granulares gruesos es pequeña [18-19, 29-37]. A pesar de lo anterior, se realizaron al inicio del programa de laboratorio, dos ensayos tipo PCC $\operatorname{con} f=0.05 \mathrm{~Hz}$ y otros dos $\operatorname{con} f=1.0$ $\mathrm{Hz}$ ( 2 y 7 , respectivamente) para medir la influencia de la frecuencia de carga sobre el comportamiento que experimenta el material granular bajo carga cíclica. En la figura 11 se observa que la influencia de la frecuencia de carga sobre la acumulación de la deformación vertical $\varepsilon_{1}{ }^{\mathrm{p}}$ es poco significativa.

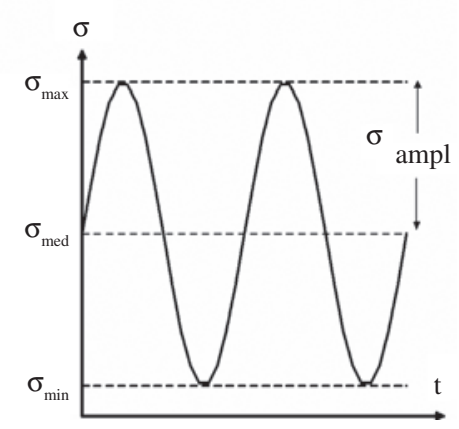

Figura 10. Notación de la distribución del esfuerzo en los ensayos cíclicos.

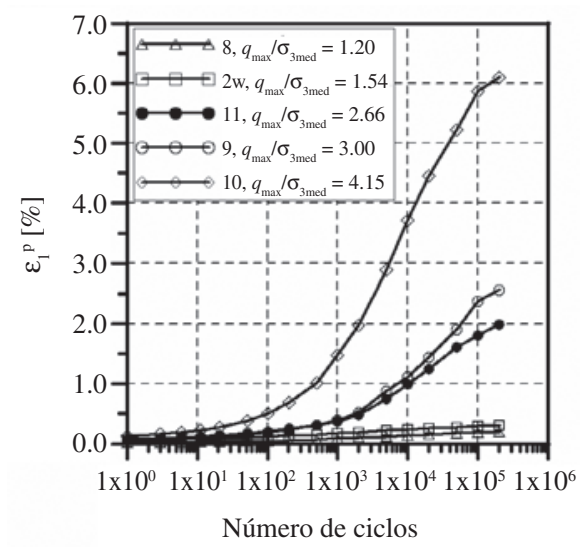

Figura $12 . \varepsilon_{1}{ }^{\mathrm{p}}$ vs. $N$ para diferentes $q_{\max } / \sigma_{3 \text { med }}$.

\section{Análisis de ensayos PCC}

En la figura 12 se observa que el valor de la deformación permanente vertical $\left(\varepsilon_{1}{ }^{\mathrm{p}}\right)$ incrementa cuando se aumenta la relación entre el esfuerzo desviador máximo y la presión media de confinamiento $q_{\max } / \sigma_{3 \mathrm{med}}$. Este tipo de deformación incrementa con el número de ciclos de carga $(N)$.

En la figura 13 se presenta la evolución de la deformación permanente de corte $\varepsilon_{\mathrm{q}}{ }^{\mathrm{p}}=2 / 3\left(\varepsilon_{1}{ }^{\mathrm{p}-} \varepsilon_{3}{ }^{\mathrm{p}}\right)$ con la componente volumétrica $\varepsilon_{\mathrm{v}}{ }^{\mathrm{p}}=\varepsilon_{1}{ }^{\mathrm{p}}+2 \varepsilon_{3}{ }^{\mathrm{p}}$. En esta figura se observa que la relación $\varepsilon_{\mathrm{q}}{ }^{\mathrm{p}} / \varepsilon_{\mathrm{v}}{ }^{\mathrm{p}}$ incrementa cuando se aumenta la pendiente promedio de la trayectoria de esfuerzo $\eta_{\text {med }}=q_{\text {med }} / p_{\text {med }}$. Es interesante observar en $\eta_{\text {med }}=0.5$ y $\eta_{\text {med }}=0.61$ (ensayos $\mathrm{N}^{\circ} 8$ y 2 , respectivamente) que la acumulación de la deformación es netamente volumétrica $\left(\varepsilon_{\mathrm{q}}{ }^{\mathrm{p}}=0, \varepsilon_{1}{ }^{\mathrm{p}} \approx \varepsilon_{3}{ }^{\mathrm{p}}\right)$. Tal vez este comportamiento se debe a que el material presenta una mayor rigidez en la dirección vertical que horizontal (debido a la forma como se compactaron las 
muestras en el laboratorio empleando el ensayo Proctor). Ensayos con trayectorias de esfuerzos adicionales a las utilizadas en este proyecto deben ser realizados con el fin de observar si el método de compactación de los especímenes influye en la dirección de la acumulación de la deformación $\varepsilon_{\mathrm{q}}{ }^{\mathrm{p}} / \varepsilon_{\mathrm{v}}{ }^{\mathrm{p}}$.

En la figura 14 se observa que la tasa de deformación disminuye con la evolución de la deformación permanente y $N$. Además esta tasa es menor cuando la relación entre $q_{\max }$ I $\sigma_{3 \mathrm{med}}$ decrece. Para los niveles de esfuerzo $q_{\max } / \sigma_{3 \mathrm{med}}=1.20$ y 1.54 se podría pensar que el material experimenta un estado "Shakedown" [15] en el cual la respuesta del material tiende a ser casi totalmente resiliente. En la figura 15 se observa que la relación de vacíos disminuye con el número de ciclos de carga hacia un valor mínimo asintótico.

\section{Análisis de ensayos PCV}

El análisis de la respuesta que experimentan materiales granulares en ensayos PCV se debe realizar de manera

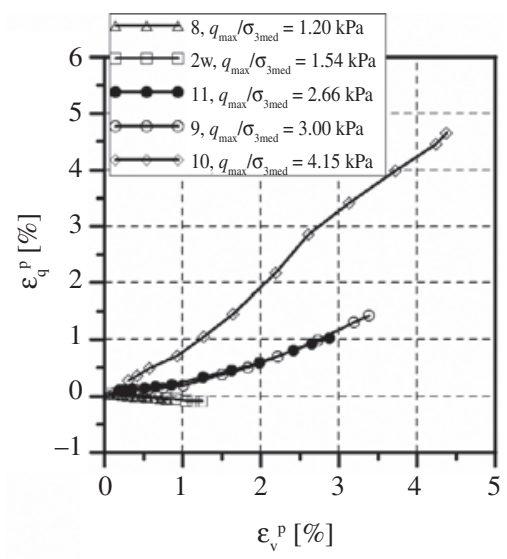

Figura 13. Deformación permanente de corte $\varepsilon_{\mathrm{q}}{ }^{\mathrm{p}}$ versus deformación permanente volumétrica $\varepsilon_{\mathrm{v}}{ }^{\mathrm{p}}$.

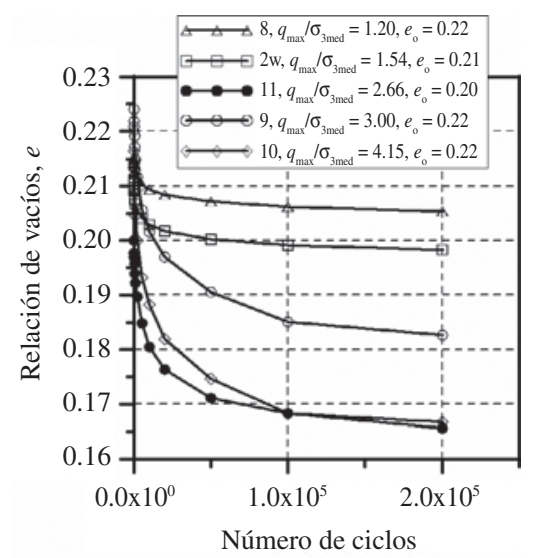

Figura 15. Evolución de la relación de vacíos $(e) \operatorname{con} N$. diferente a como fue realizado para el caso de los PCC, ya que en los PCV la presión de confinamiento $\sigma_{3}$ no permanece constante durante el ensayo (varía desde un valor mínimo $\sigma_{3 \min }$ a uno máximo $\sigma_{3 \max }$ ). En [38], basados en ensayos PCV sobre dos materiales granulares distintos (denominados Poulmarch y Sorèze), observaron que la deformación vertical permanente incrementa cuando se aumenta la relación entre el esfuerzo desviador y la presión media $(q / p)$. Además reportaron un incremento en la deformación con un aumento en la presión media p. Similar observación fue reportada por Habiballah y Chazallon [39] sobre una arena con $d_{\max }=4 \mathrm{~mm}$. Basado en lo anterior se propone realizar el análisis de los ensayos PCV graficando la evolución de la deformación permanente con la longitud de la trayectoria de esfuerzo $L=\sqrt{\left(q_{\max }-q_{\min }\right)^{2}+\left(p_{\max }-p_{\min }\right)^{2}}$. En la

figura 16 se observa que la deformación permanente vertical incrementa proporcionalmente con la magnitud del esfuerzo cíclico $L$ y con el número de ciclos de carga $(N)$.

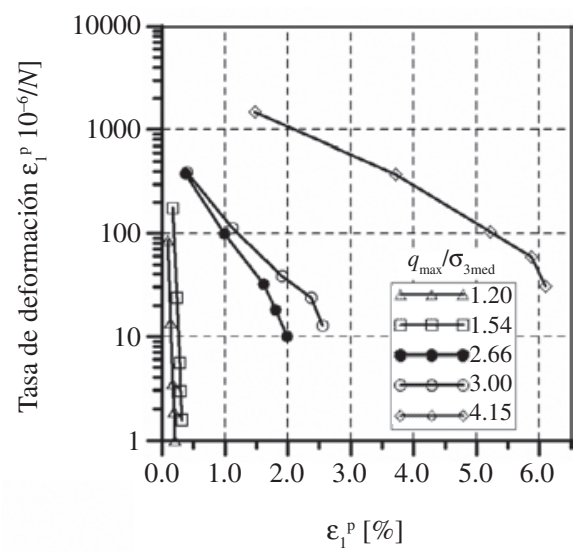

Figura 14. Tasa de deformación vs. $\varepsilon_{1}{ }^{\mathrm{p}}$.

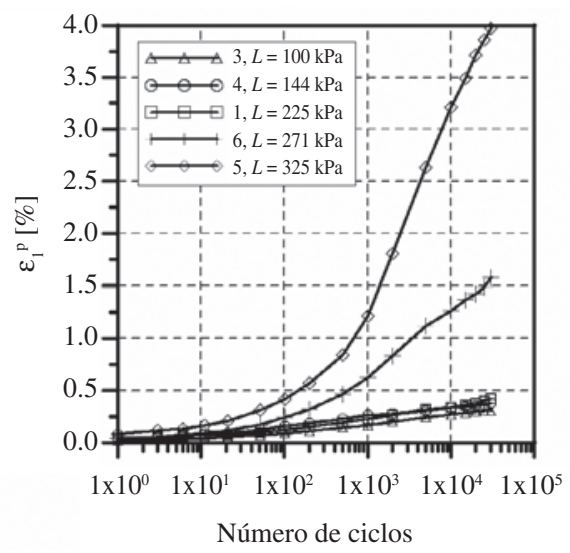

Figura $16 . \varepsilon_{1}{ }^{\mathrm{p}}$ vs. $N$ para diferentes $L$. 
De manera similar a los ensayos PCC, la tasa de deformación vertical disminuye con la evolución de la acumulación de la deformación vertical, y esta tasa es menor cuando $L$ decrece (figura 17). Además en la figura 18 se observa que la relación de vacíos disminuye con $N$ hacia un valor mínimo asintótico.

\section{Comparación ensayos PCC y PCV}

La comparación de los ensayos PCC y PCV es presentada en la figura 19 (la cual es similar a la figura 3 de los resultados reportados por Brown y Hyde [20]). En la figura se presentan los resultados para $N=10^{3}, 5 \times 10^{3}, 10^{4}, 2 \times 10^{4}$. Se observa que el comportamiento que experimentó el material es dependiente del valor de $\eta_{\text {ampl}}$, por lo tanto suponer que la deformación permanente no se afecta por el tipo de ensayo como lo aseguran Brown y Hyde [20] no es cierto. Las figuras 20a-e, y 21a-e, ayudan a entender mejor la anterior afirmación.

En las figuras $20 \mathrm{a}$ y b $\left(\eta_{\mathrm{ampl}}=0.75\right)$ la deformación permanente vertical fue mayor cuando se realizaron

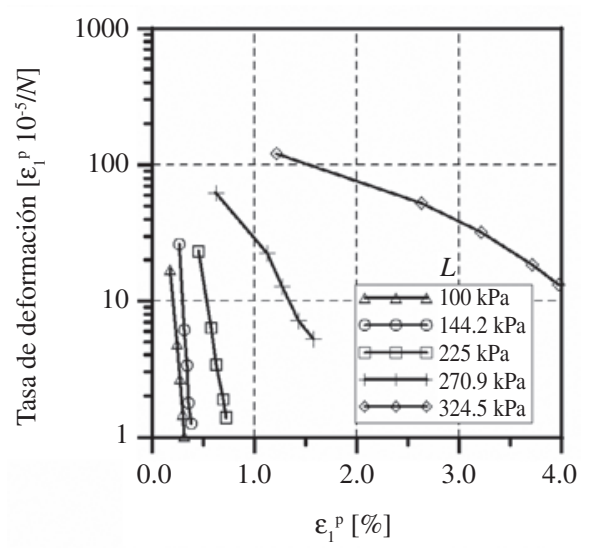

Figura 17. Tasa de deformación vs. deformación vertical para los ensayos PCV. ensayos PCV. Este comportamiento cambió cuando se utilizaron trayectorias con $\eta_{\mathrm{ampl}}=1.5$ (figuras $20 \mathrm{c} \mathrm{y} \mathrm{d}$ ). La acumulación de la deformación vertical en ensayos PCC con $\eta_{\text {ampl }}=1.5$ es mayor cuando la amplitud del esfuerzo es pequeña. Para el caso de $\eta_{\text {ampl }}=1.5$ y altos valores de amplitud de esfuerzo la acumulación de la deformación en ensayos PCC y PCV es similar. Tal vez este cambio de comportamiento en $\eta_{\text {ampl }}=0.75 \mathrm{y}$ $\eta_{\mathrm{ampl}}=1.5$ se debe a que para $\eta_{\mathrm{ampl}}=1.5$ la distancia entre el $q_{\max }$ y el esfuerzo desviador necesario para llevar a la falla al material $q_{\text {falla }}$ en los ensayos PCC es más pequeña en comparación con $\eta_{\mathrm{ampl}}=0.75$. Además de las figuras 14 y 17 se puede deducir que la variación de $\eta_{\text {ampl }}$ en ensayos PCC genera un incremento mayor de la tasa de deformación en comparación con los ensayos PCV. Para el caso de $\eta_{\text {ampl }}=1.125$ la tendencia fue a experimentar valores similares de deformación vertical en ambos ensayos. Existe entonces un valor de $\eta_{\text {ampl }}$ para el cual el tipo de ensayo no influye sobre el valor de la acumulación de la deformación vertical.

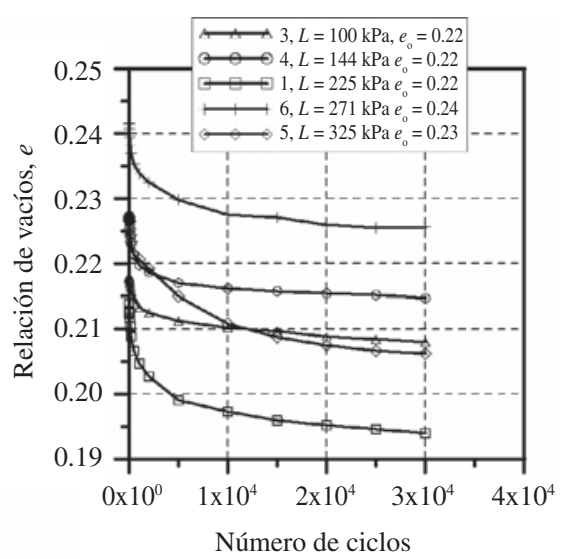

Figura 18. Evolución de la relación de vacíos con $N$.

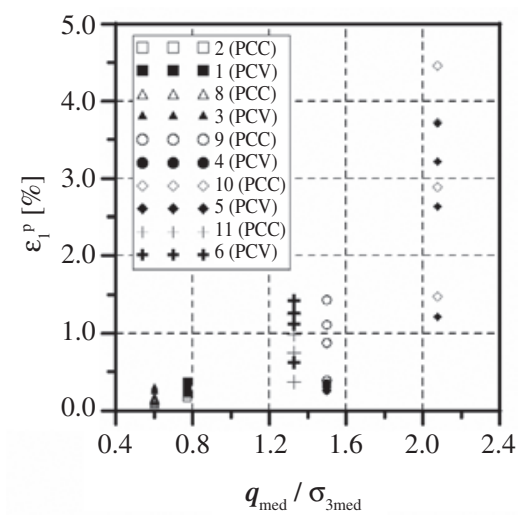

Figura 19. Influencia de $\mathrm{q}_{\text {med }} / \sigma_{3 \text { med }}$ sobre la deformación vertical para diferentes $N$. 


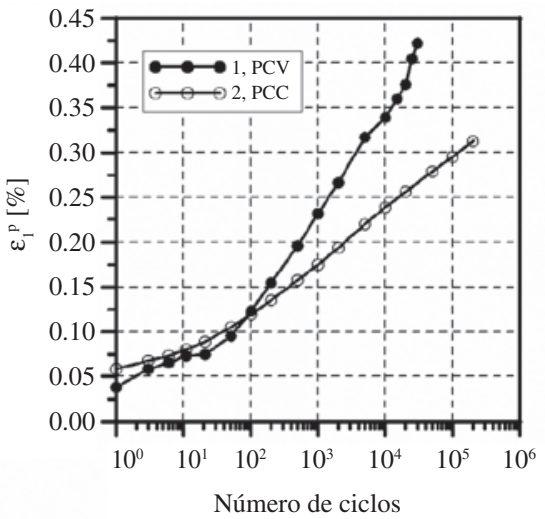

(A)

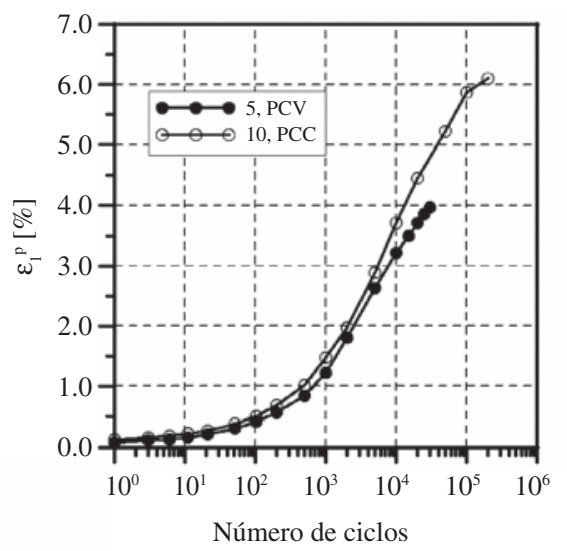

(d)

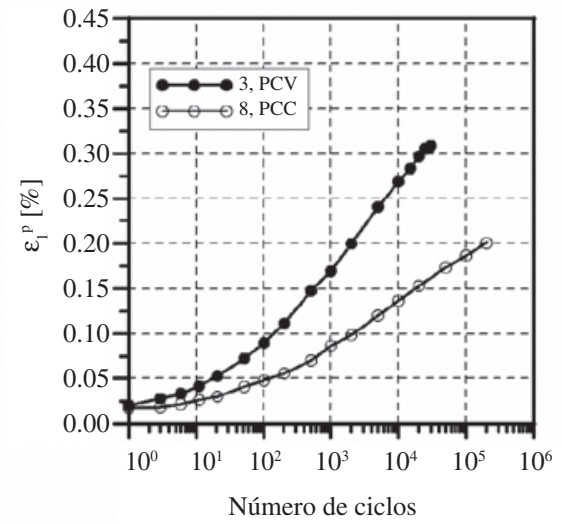

(B)

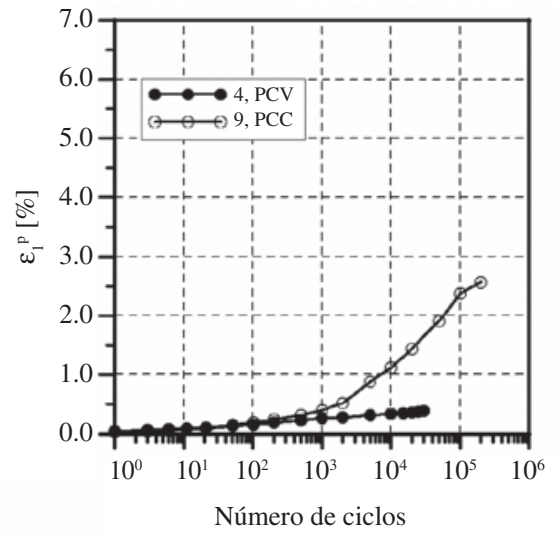

(C)

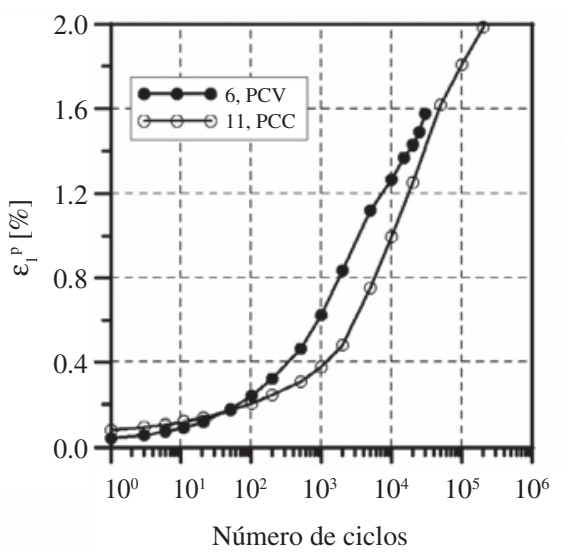

(e)

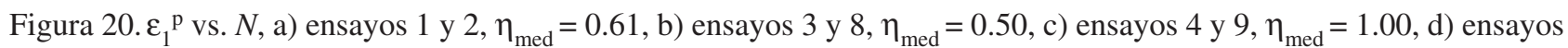
5 y $10, \eta_{\text {med }}=1.23$, e) ensayos 6 y $11, \eta_{\text {med }}=0.92$.

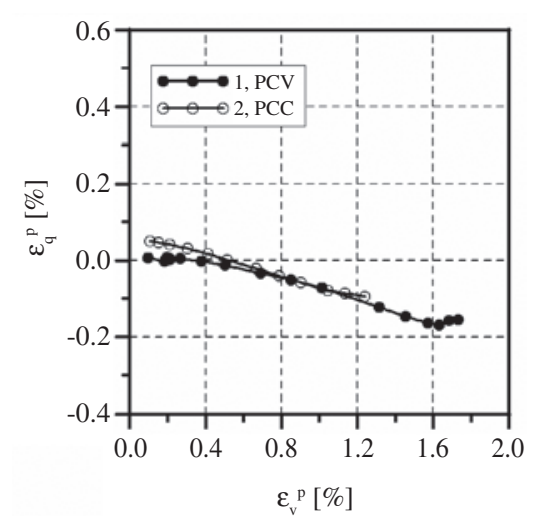

(a)

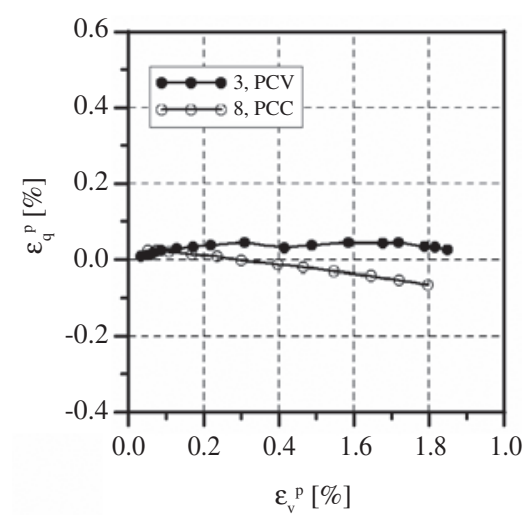

(b)

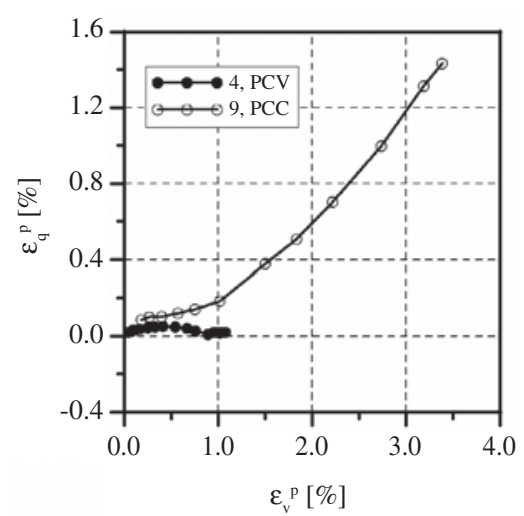

(c)

Figura 21. $\varepsilon_{\mathrm{q}}^{\mathrm{p}}$ vs. $\varepsilon_{\mathrm{v}}^{\mathrm{p}}$, a) ensayos 1 y $2, \eta_{\text {med }}=0.61, \mathrm{~b}$ ) ensayos 3 y $8, \eta_{\text {med }}=0.50$, c) ensayos 4 y $9, \eta_{\text {med }}=1.00$. 


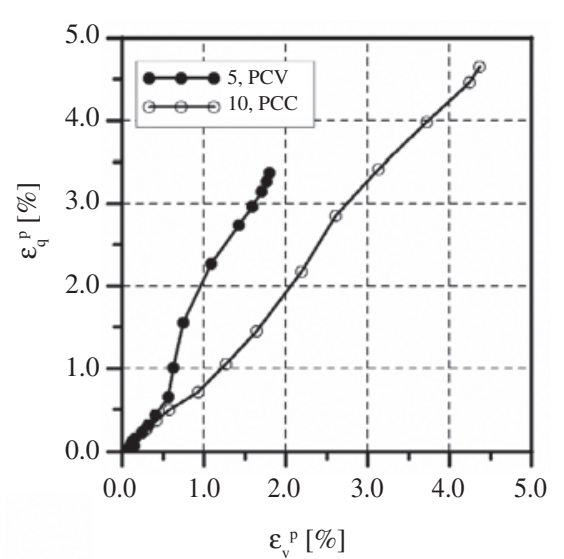

(d)

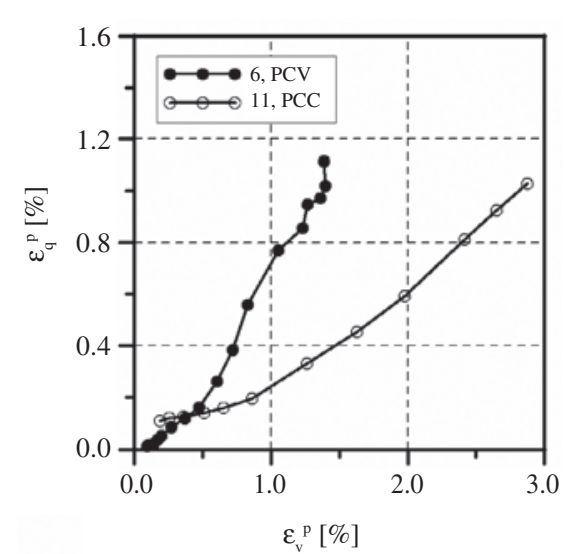

(e)

Figura 21.d) ensayos 5 y $10, \eta_{\text {med }}=1.23$, e) ensayos 6 y $11, \eta_{\text {med }}=0.92$.

Para $\eta_{\mathrm{ampl}}=0.75$ la dirección de la acumulación de la deformación $\varepsilon_{\mathrm{q}}{ }^{\mathrm{p}} / \varepsilon_{\mathrm{v}}{ }_{\mathrm{v}}^{\mathrm{p}}$ es similar en los ensayos PCV y PCC (figuras $21 \mathrm{a}$, b). En $\eta_{\mathrm{ampl}}=1.125$ y 1.50 el material experimenta mayor $\varepsilon_{\mathrm{q}}{ }^{\mathrm{p}} / \varepsilon_{\mathrm{v}}{ }^{\mathrm{P}}$ en ensayos PCV cuando la amplitud del esfuerzo es alta (figuras $21 \mathrm{~d}$, e). Cuando $\eta_{\mathrm{ampl}}=1.50$ y la amplitud del esfuerzo es pequeña, la dirección de la acumulación es mayor en ensayos PCC (figura 21c).

\section{CONCLUSIONES}

En la presente investigación se realizaron diferentes ensayos triaxiales cíclicos con presión de confinamiento constante (PCC) y variable (PCV) con el fin de comparar la respuesta que experimenta un material granular en ambos ensayos cuando el promedio de esfuerzos ( $\left.p_{\text {med }}, q_{\text {med }}\right)$ y la amplitud de los esfuerzos desviadores $\left(q_{\text {ampl }}\right)$ son los mismos. El estudio demuestra que, en general, el material experimentó diferente acumulación de la deformación en ambos ensayos. Sin embargo, bajo algunas trayectorias de esfuerzos, las componentes verticales y volumétricas de acumulación de la deformación fueron iguales (por ejemplo la deformación vertical cuando las amplitudes de carga son altas).

Para el diseño de pavimentos, la deformación permanente vertical es la componente más importante de la deformación. Para pequeños valores de $\eta_{\text {ampl }}=q_{\text {ampl }} / p_{\text {ampl }}=0.75$, este tipo de deformación fue mayor en los ensayos PCV que en los correspondientes ensayos PCC, independientemente de la amplitud de esfuerzo aplicado al material. Cuando se aplicaron valores intermedios de $\eta_{\mathrm{ampl}}=1.125 \mathrm{la}$ deformación permanente en ambos ensayos fue similar. De la misma forma, el material experimentó igual acumulación de la deformación vertical cuando se aumentó la inclinación de la trayectoria $\left(\eta_{\mathrm{ampl}}=1.5\right)$ y se combinaba con amplitudes de esfuerzo altas, pero para el caso de amplitudes pequeñas, el material desarrolló mayor deformación en los ensayos PCC que en los PCV. De lo anterior se concluye que dependiendo de las amplitudes y de la inclinación de las trayectorias de esfuerzo, el material experimenta diferentes respuestas esfuerzo-deformación.

En un pavimento los valores de $\eta$ por lo general son pequeños. En este rango de valores de esfuerzo, los ensayos PCC subestiman la deformación que experimenta el material granular en comparación con los ensayos PCV. Sin embargo, es importante tener en cuenta que ambos ensayos no pueden reproducir la componente cíclica de esfuerzo de corte y por tal motivo es necesario realizar estudio adicionales en donde se pueda comparar la influencia de las tres componentes (vertical, horizontal y de corte) aplicadas simultáneamente al material.

\section{REFERENCIAS}

[1] R. Bonaquist. "Summary of Pavement Performance Test Using the Accelerated Loading Facility, 1986 - 1990". Transportation Research Record, Transportation Research Board. Washington, D. C. $\mathrm{N}^{\mathrm{o}} 1354$, pp. 74-85. 1992.

[2] N.W. Lister. "The Transient and Long Term Performance of Pavements in Relation to Temperature". Proc. 3rd. Int. Conf. on Structural Design of Asphalt Pavements. University of Michigan, Ann Arbor. 1972.

[3] A.C. Collop, D. Cebon and M.S.A. Hardy. "Viscoelastic Approach to Rutting in Flexible Pavements". Journal of Transportation Engineering. Vol. $121 \mathrm{~N}^{\circ}$ 1, pp. 82-93. 1995. 
[4] P.H. Little. "The Design of Unsurfaced Roads Using Geosynthetics". Tesis para optar al grado de doctor de Filosofía. Universidad de Nottingham, Nottingham, U.K. 1993.

[5] S.F. Brown. "Soil Mechanics in Pavement Engineering. The 36th Rankine Lecture of the British Geotechnical Society". Géotechnique. Vol. $46 \mathrm{~N}^{\mathrm{o}} 3$, pp. 383-426. 1996.

[6] A.R. Archilla and S. Madanat. "Estimation of Rutting Models by Combining Data from Different Sources". Journal of Transportation Engineering. Vol. $127 \mathrm{~N}^{\circ}$ 5, pp. 379-389. 2002.

[7] S. Erlingsson. "Mechanistic Pavement Design Methods - A Road to Better Understanding of Pavement Performance”. Via Nordica 2004 NRA's 19th Road Congress, C8: Berekraftige vegkonstruksjonar, p. 8. 2004.

[8] S. Erlingsson and T. Ingason. "Performance of Two Thin Pavement Structures During Accelerated Pavement Testing Using a Heavy Vehicle Simulator". 2nd International Conferences on Accelerated Pavement Testing, University of Minnesota, $\mathrm{p}$. 19. 2004.

[9] T. Ingason, L.G. Wiman and H. Haraldsson. "HVS - Testing of Iceland Low Volume Road Structures". ISAP - 9th International Conference on Design of Asphalt Pavements, p. 13. 2002.

[10] B.D. Pidwerbesky. "Fundamental Behaviour of Unbound Granular Pavements Subjected to Various Loading Conditions and Accelerated Trafficking". Tesis para optar al grado de doctor de Filosofía. Universidad de Canterbury. Christchurch, New Zealand. 1996.

[11] F. Lekarp, I.R. Richardson and A. Dawson. "Influences on Permanent Deformation Behavior of Unbound Granular Materials". Transportation Research Record, Transportation Research Board, Washington D. C. N 1547, pp. 68-75. 1996.

[12] J. Uzan. "Granular Material Characterization for Mechanistic Pavement Design”. Journal of Transportation Engineering, Vol. 125, pp. 108-113. 1999.

[13] F. Lekarp, I. Ulf and A.R. Dawson. "State of the art. I: Resilient Response of Unbound Aggregates". Journal of Transportation Engineering, Vol. 126 No 1, pp. 66-75. 2000.
[14] I.F. Collins and M. Boulbibane. "Geomechanical Analysis of Unbound Pavements Based on Shakedown Theory". Journal of Geotechnical and Geoenvironmental Engineering, Vol. 126, pp. 50-59. 2000.

[15] S. Werkmeister. "Permanent Deformation Behaviour of Unbound Granular Materials in Pavement Constructions". Tesis para optar al grado de doctor en Filosofía. University of Technology, Dresden. 2003.

[16] S. Werkmeister, R. Numrich, A. Dawson and F. Wellner. "Deformation Behaviour of Granular Materials under Repeated Dynamic Load". Journal of Environmental Geomechanics - Monte Veritá, pp. 215-223. 2002.

[17] A.R. Dawson. "Cumulative Damage and its Applicability to Low Volume Road Pavements". Internationales Kolloquium Prüfung, Bewertung und Bemessung von Straßenbefestigungen, Technische Universitat Dresden. April 3-4, 2003.

[18] J.J.Allen and M.R. Thompson. "Resilient Response of Granular Materials Subjected to Time-Dependent Lateral Stresses". Transportation Research Record, Transportation Research Board, Washington, D. C. No 510, pp. 1-13. 1974.

[19] S.F. Brown. "Repeated Load Testing of a Granular Material". Journal of the Geotechnical Engineering Division. Vol. 100 N $^{\text {7 }}$, pp. 825-841. 1974.

[20] S.F. Brown and A.F.L. Hyde. "Significance of Cyclic Confining Stress in Repeated-load Triaxial Testing of Granular Material". Transportation Research Record, Transportation Research Board, Washington, D. C. No 537, pp. 49-58. 1975.

[21] INVIAS - Instituto Nacional de Vías. "Especificaciones Generales para Construcción de Carreteras”. Bogotá D.C., Colombia. 2002.

[22] F. Lekarp, I. Ulf and A.R. Dawson. "State of the art II: Permanent Strain Response of Unbound Aggregates". Journal of Transportation Engineering. Vol. $126 \mathrm{~N}^{\mathrm{o}}$ 1, pp. 76-83. 2000.

[23] H.A. Rondón. "Comportamiento de un material granular en ensayos triaxiales cíclicos con presión de confinamiento constante (PCC) y variable (PCV)". Tesis para optar al grado de doctor en Ingeniería. Universidad de los Andes. Abril, 2008. 
[24] H.A. Rondón y F.A Reyes. "Comportamiento de materiales granulares en pavimentos flexibles: estado del conocimiento". Ed. Universidad Católica de Colombia y Pontificia Universidad Javeriana, p. 119. Bogotá D.C., Colombia. 2008.

[25] INVIAS - Instituto Nacional de Vías. "Normas de Ensayos de Materiales para Carreteras". Bogotá D.C., Colombia. 2002.

[27] H.A. Rondón, T. Wichtmann, Th. Triantafyllidis and A. Lizcano. "Hypoplastic material constants for a well-graded granular material (UGM) for base and subbase layers of flexible pavements". Acta Geotechnica. Vol. 2 № 2, pp. 113-126. 2007.

[28] A. Niemunis, T. Wichtmann and Th. Triantafyllidis. "On the definition of the fatigue loading for sand". In International Workshop on Constitutive Modelling - Development, Implementation, Evaluation, and Application, Hong Kong. 2007.

[29] T.L. Youd. "Compaction of Sands by Repeated Shear Straining". Journal of the Soil Mechanics and Foundations Division, ASCE, 98 (SM7), pp. 709-725. 1972.

[30] I.V. Kalcheff and R.G. Hicks. "A Test Procedure for Determining the Resilient Properties of Granular Materials". Journal of Testing and Evaluation, JTEVA. Vol. 1 No 6, pp. 472-479. 1973.

[31] T. Kokusho, T. Hara and R. Hiraoka. "Undrained Shear Strength of Granular Soils with Different Particle Gradations". Journal of Geotechnical and Geoenvironmental Engineering, ASCE. Vol. 130 $\mathrm{N}^{\circ}$ 6, pp. 621-629. 2004.

[32] C. Rada and W.M. Witczak. "Comprehensive Evaluation of Laboratory Resilient Moduli Results for Granular Materials”. Transportation Research Record, Transportation Research Board, Washington D. C., No 810, pp. 23-33. 1981.
[33] J.R. Boyce. "The Behaviour of a Granular Material under Repeated Loading". Tesis para optar al grado de doctor de Filosofía. Universidad de Nottingham. Nottingham, U.K. 1976.

[34] M.J. Shenton. "Deformation of Railway Ballast under Repeated Loading Conditions". Railroad Track Mechanics and Technology. Pergamon Press, pp. 405-425. 1978.

[35] H.L. Theyse. "Stiffness, Strength, and Performance of Unbound Aggregate Materials: Application of South African HVS and Laboratory Results to California Flexible Pavements". Report produced under the auspices of the California Partnered Pavement Research Program for the California Department of Transportation. University of California, p. 76. 2002.

[36] N.H Thom and S.F. Brown. "Effect of Moisture on the Structural Performance of a Crushed-Limestone Road Base". Transportation Research Record. Transportation Research Board. Washington D. C. No 1121, pp. 50-56. 1987.

[37] T. Wichtmann. "Explicit Accumulation Model for Non-cohesive Soils under Cyclic Loading". Tesis para optar al grado de doctor en Filosofía. Des Institutes für Grundbau und Bodenmechanik der Ruhr-Universität Bochum. 2005.

[38] G. Gidel, P. Hornych, J. Chauvin, D. Breysse and A. Denis. "A New Approach for Investigating the Permanent Deformation Behavior of Unbound Granular Material Using the Repeated Load Triaxial Apparatus". Bulletin Des Laboratoires Des Ponts et Chaussées, pp. 5-21. 2001.

[39] T. Habiballah and C. Chazallon. "An Elastoplastic Model Based on the Shakedown Concept for Flexible Pavements Unbound Granular Materials". Int. J. Numer. Anal. Meth. Geomech. Vol. 29, pp. 577-596. 2005. 